\title{
Advanced gastric cancer with abdominal wall invasion treated with curative resection after chemotherapy: a case report
}

Naohiko Nakamura*, Shinichi Kinami, Jun Fujita, Daisuke Kaida, Yasuto Tomita, Takashi Miyata, Hideto Fujita, Nobuhiko Ueda and Hiroyuki Takamura

\begin{abstract}
Introduction: In patients with gastric cancer, $6-27 \%$ of patients are diagnosed with $\mathrm{T} 4 \mathrm{~b}$ disease that invades adjacent organs, and curative resection can improve the prognosis of these patients.

Case presentation: A 70-year-old Japanese man presented with an abdominal tumor and was diagnosed with advanced gastric cancer (L-Circ type 3 T4b N2 MO H0 stage IVA, based on the 15th edition of the Japanese Classification of Gastric Carcinoma) with extensive abdominal wall invasion. We performed open gastrojejunal bypass for gastric obstruction and initiated a chemotherapeutic regimen comprising S-1 (120 mg/day) and oxaliplatin (100 mg/ $\mathrm{m}^{2}$ ). Upper gastrointestinal endoscopy performed after the administration of six courses of the S-1 and oxaliplatin regimen revealed a persistent primary lower gastric wall lesion; however, the diameter of the abdominal wall invasion and metastatic lymph nodes was significantly reduced, in addition to decreased serum carcinoembryonic antigen and carbohydrate antigen 19-9 levels. Subsequently, the patient underwent distal gastrectomy with D2 lymphadenectomy combined with transverse colon and abdominal wall resection. We performed radical en bloc resection and achieved a tumor-free resection margin. Simple abdominal wall closure was performed without mesh or musculocutaneous flap placement. Histopathological examination of the resected tumor specimen showed direct invasion of the mesocolon and rectus abdominis muscle. The patient was postoperatively diagnosed with $L$ Gre-Ant type5 T4b (SI: rectus abdominis muscle) N2 PMO DMO Stage IIIA RO Grade 2a gastric cancer based on histopathological findings and received S-1 as adjuvant chemotherapy, 2 months postoperatively. No recurrence was detected 6 months postoperatively.
\end{abstract}

Conclusions: We report a case of advanced gastric cancer with extensive abdominal wall invasion that was successfully treated with gastrectomy combined with resection of adjacent organs showing tumor invasion after effective systemic chemotherapy. A therapeutic approach comprising curative surgery combined with perioperative chemotherapy is useful in patients with T4b gastric cancer.

Keywords: Gastric cancer, T4b, Abdominal wall invasion, Gastrectomy, Case report

*Correspondence: n1982422@kuhp.kyoto-u.ac.jp Department of Surgical Oncology, Kanazawa Medical University, 1-1 Daigaku, Uchinada, Kahoku, Ishikawa 920-0293, Japan

\section{Background}

Gastric cancer (GC) is the fifth most common malignancy and the third most common cause of cancer mortality worldwide [1]. Although early GC is curable, advanced-stage disease is associated with poor survival, and curative treatment consists of gastrectomy with perioperative chemotherapy $[2,3]$. Based on the 8 th edition original author(s) and the source, provide a link to the Creative Commons licence, and indicate if changes were made. The images or other third party material in this article are included in the article's Creative Commons licence, unless indicated otherwise in a credit line to the material. If material is not included in the article's Creative Commons licence and your intended use is not permitted by statutory regulation or exceeds the permitted use, you will need to obtain permission directly from the copyright holder. To view a copy of this licence, visit http://creativecommons.org/licenses/by/4.0/. The Creative Commons Public Domain Dedication waiver (http://creativeco mmons.org/publicdomain/zero/1.0/) applies to the data made available in this article, unless otherwise stated in a credit line to the data. 
of the International Union Against Cancer TNM classification and the 15th edition of the Japanese Classification of Gastric Carcinoma, advanced GC that invades adjacent organs is classified as T4b GC $[4,5]$. Notably, $6-27 \%$ of patients with GC are diagnosed with T4b disease [6-11], and curative resection can improve the prognosis of these patients [6-9]. Curative resection is indicated in $\leq 50 \%$ of patients with T4b GC because these patients invariably present with peritoneal dissemination and distant metastasis that frequently complicate T4b GC [12-14]. Therefore, accurate diagnosis of progressive disease and preoperative administration of effective chemotherapy are important to achieve curative resection in patients with T4b GC.

We present a case of advanced GC with extensive abdominal wall invasion that was successfully treated with gastrectomy combined with adjacent organ resection after effective systemic chemotherapy.

\section{Case presentations}

A 70-year-old Japanese man (height $178 \mathrm{~cm}$, weight $54.0 \mathrm{~kg}$, and Eastern Cooperative Oncology Group Performance Status score 0) presented with an abdominal tumor and suspected advanced GC. He had previously undergone an operation for ileus, and his family history was not significant. A contrast-enhanced computed tomography scan $(\mathrm{eCT})$ revealed extensive abdominal wall invasion by the main gastric tumor in addition to enlarged peritumoral lymph nodes; however, no lung and liver metastases were detected (Fig. 1). Upper gastrointestinal endoscopy revealed a circumferential Borrmann type III tumor at the lower gastric wall with tumor-induced stenotic obstruction (Fig. 2a). Biopsy of the gastric tumor revealed a moderately differentiated tubular adenocarcinoma. Immunohistochemical evaluation showed a human epidermal growth factor
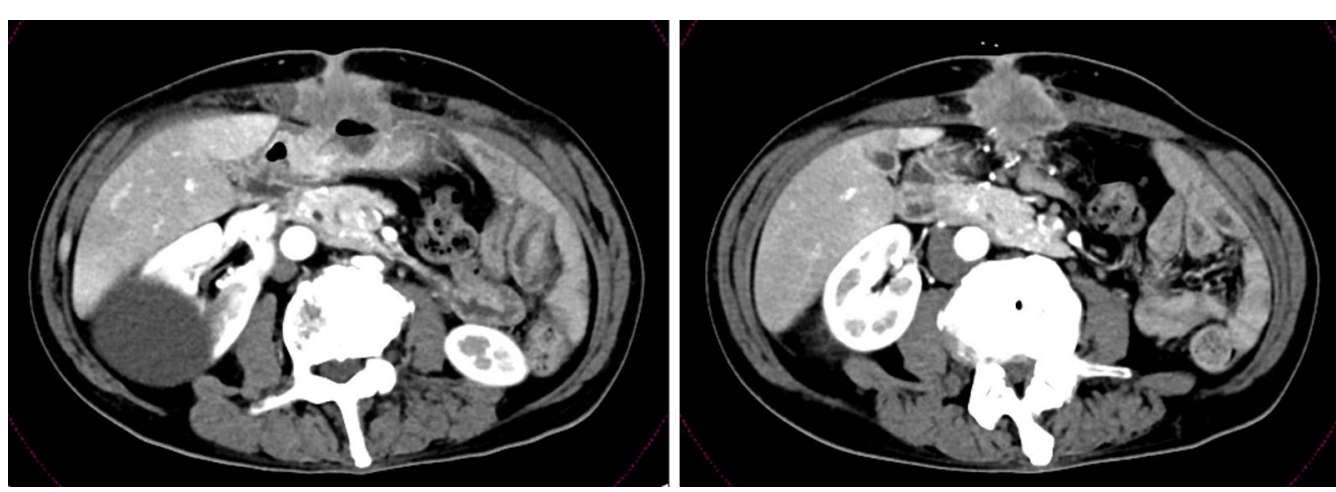

Fig. 1 Pretreatment contrast-enhanced computed tomography scan showing the primary gastric tumor with massive invasion of the abdominal wall and enlarged peritumoral lymph nodes
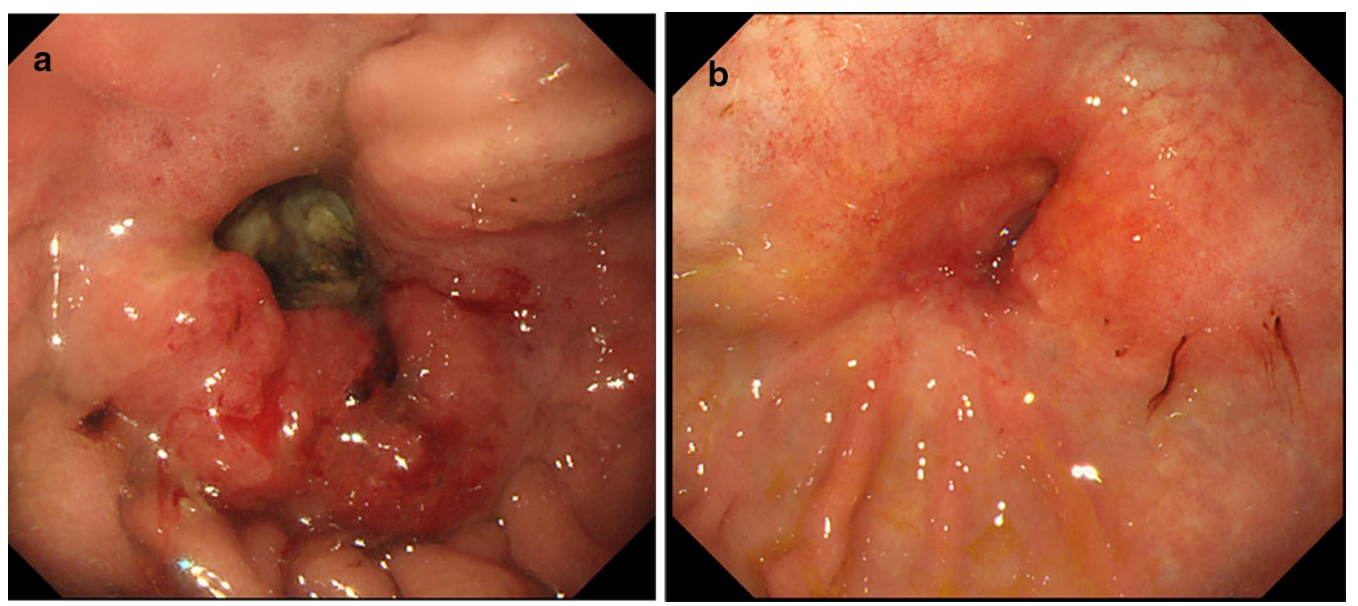

Fig. 2 Upper gastrointestinal endoscopy image obtained before treatment (a) and image obtained after chemotherapy (b) showing the following findings: a Borrmann type III tumor with stenosis observed at the lower gastric wall. b A persistent primary lesion visualized at the lower gastric wall; however, the lesion has regressed in size 
receptor 2-negative lesion, and the patient was clinically diagnosed with L-Circ type 3 T4b N2 M0 H0 stage IVA GC (based on the 15th edition of the Japanese Classification of Gastric Carcinoma [5]). We performed open gastrojejunal bypass for gastric obstruction and confirmed extensive abdominal wall invasion of the tumor and absence of peritoneal dissemination (Fig. 3). Then, we initiated the SOX chemotherapeutic regimen comprising S-1 (120 mg/day, day 1 to day 14$)$ and oxaliplatin $\left(100 \mathrm{mg} / \mathrm{m}^{2}\right.$, every 21 days). Although neutropenia (Grade 3, based on the National Cancer Institute Common Terminology Criteria for Adverse Events version 3.0) occurred as an adverse event associated with SOX therapy, the diameter of the abdominal wall invasion and metastatic lymph nodes was reduced after the administration of four courses of the SOX regimen. Therefore, we administered six courses of the SOX regimen. Upper gastrointestinal endoscopy revealed a persistent primary lesion of the lower gastric wall (Fig. 2b);

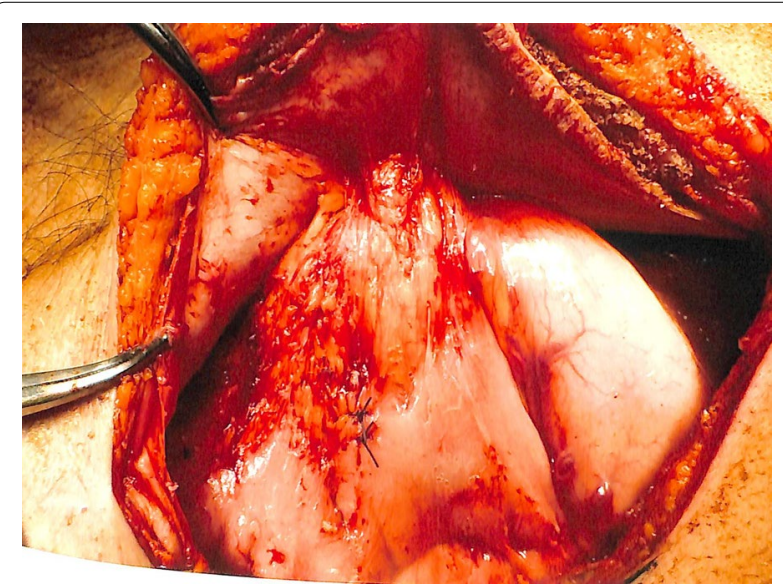

Fig. 3 Intraoperative finding during open gastrojejunal bypass however, the diameter of the abdominal wall invasion and metastatic lymph nodes was significantly reduced in addition to decreased serum carcinoembryonic antigen and cancer antigen 19-9 levels (Fig. 4). Notably, eCT performed after six courses of the SOX regimen revealed a partial response to therapy based on The Response Evaluation Criteria in Solid Tumors, version 1.1 guidelines. Subsequently, we performed distal gastrectomy with D2 lymphadenectomy combined with transverse colon and abdominal wall resection because we observed direct tumor invasion of the mesocolon and rectus abdominis muscle. We performed radical en bloc resection and achieved a tumor-free resection margin. Although the tumor invaded the rectus abdominis muscle for which we performed abdominal wall resection, the abdominal wall defect was closed using simple sutures without mesh or musculocutaneous flap placement. Based on histopathological examination, the patient was postoperatively diagnosed with L Gre-Ant yp-TRype5 T4b (SI: rectus abdominis muscle) N2 PM0 DM0 Stage IIIA R0 Grade 2a GC (according to the 15th edition of the Japanese Classification of Gastric Carcinoma [5]) and received S-1 as adjuvant chemotherapy, 2 months postoperatively. No recurrence was detected on 6-month postoperative eCT.

\section{Discussion and conclusions}

Curative resection is the mainstay of treatment for patients with T4b GC [6-9]. However, this treatment strategy remains controversial because T4b GC is a heterogeneous and multifactorial disease. In this case, we administered systemic chemotherapy before surgical resection to ensure curative resection by minimizing invasion of adjacent organs, particularly the abdominal wall. Reportedly, the 5-year survival rate in patients with T4b GC who undergo curative resection is $20-32 \%$,
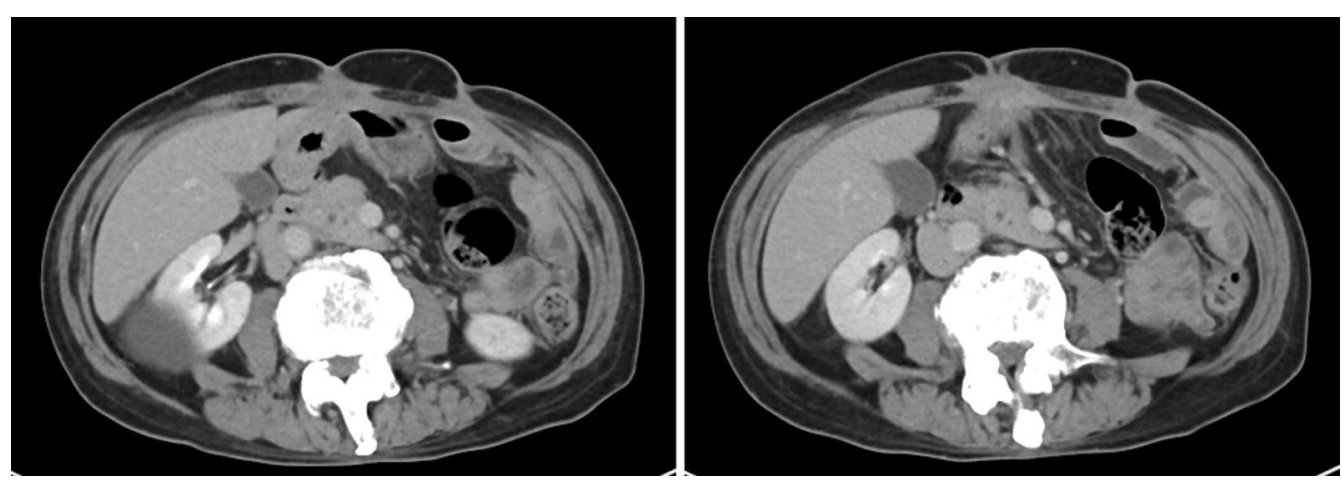

Fig. 4 Contrast-enhanced computed tomography scan obtained after six courses of the SOX regimen showing significant reduction in the diameter of the abdominal wall invasion and metastatic lymph nodes. SOX regimen: chemotherapeutic regimen comprising S-1 (120 mg/day) and oxaliplatin $\left(100 \mathrm{mg} / \mathrm{m}^{2}\right)$ 
which is significantly higher than the survival rate after noncurative resection (7-9\%) $[13,14]$. Studies have reported that patients with distant metastases showed poor prognosis even after undergoing resection of adjacent organs showing tumor invasion [12]. The pancreas, mesocolon, liver, transverse colon, adrenal glands, and spleen are most commonly invaded (based on macroscopic appearance) [10]. In this case, the tumor macroscopically invaded the mesocolon and abdominal wall. Although curative resection is possible without administration of preoperative chemotherapy, an effective response to preoperative chemotherapy contributes to successful radical en bloc resection and also improves postoperative prognosis. In this patient, the final histopathological findings revealed lymph node metastasis (stage N2). Previous studies have reported a correlation between advanced stage lymph node disease and poor prognosis after curative resection in patients with $\mathrm{T} 4 \mathrm{~b}$ GC $[15,16]$. Therefore, preoperative chemotherapy may be important for patients with T4b GC with advancedstage lymph node disease. Moreover, administration of preoperative chemotherapy significantly reduced the diameter of the abdominal wall invasion; therefore, the abdominal wall defect could be closed with simple sutures without mesh or musculocutaneous flap placement. Tumor stage, expected survival, and postresection quality of life should be carefully evaluated for optimal planning to minimize the extent of surgery.

In conclusion, we report a case of advanced GC with extensive abdominal wall invasion that was successfully treated with gastrectomy combined with resection of adjacent organs showing tumor invasion after effective systemic chemotherapy. In our view, a therapeutic approach comprising curative surgery combined with perioperative chemotherapy is useful in patients with T4b GC.

\section{Abbreviations \\ GC: Gastric cancer; TNM: Tumor node metastasis; eCT: Contrast-enhanced computed tomography scan; SOX: Chemotherapy combining S-1 and oxaliplatin.}

\section{Acknowledgements}

Not applicable.

\section{Authors' contributions}

NN reported the case and wrote the manuscript. SK, YF, SM, JF, DK, YT, TM, HF, $\mathrm{NU}$, and $\mathrm{HT}$ were involved in treating the patient. SK and $\mathrm{HT}$ participated in critically revising the manuscript. All authors have read and approved the final manuscript.

\section{Funding}

None.

\section{Availability of data and materials}

All data are available without restriction. Researchers can obtain data by contacting the corresponding author.

\section{Declarations}

\section{Ethics approval and consent to participate}

This study was approved by the Medicine Ethics Committee of Kanazawa Medical University. We obtained informed consent from the patient verbally.

\section{Consent for publication}

Written informed consent was obtained from the patient for publication of this case report and any accompanying images. A copy of the written consent is available for review by the Editor-in-Chief of this journal.

\section{Competing interests}

The authors declare no competing interest in connection with this study.

Received: 8 June 2020 Accepted: 24 March 2021

Published online: 09 May 2021

\section{References}

1. Ferlay J, Soerjomataram I, Dikshit R, Eser S, Mathers C, et al. Cancer incidence and mortality worldwide: sources, methods and major patterns in GLOBOCAN 2012. Int J Cancer. 2015;136:E359-386.

2. Cunnimgham D, Allum WH, Stenning SP, Thompson JN, Van de Velde $C J$, et al. MAGIC Trial Participants. Perioperative chemotherapy versus surgery alone for resectable gastroesophageal cancer. N Engl J Med, 2006; 355: $11-20$.

3. Brenkman HJ, Haverkamp L, Ruurda JP, van Hillegersberg R. Worldwide practice in gastric cancer surgery. World J Gastroenterol. 2016;22:4041-8.

4. In H, Solsky I, Palis B, Langdon-Embry M, Ajani J, et al. Validation of the 8th edition of the AJCC TNM Staging System for Gastric Cancer using the National Cancer Database. Ann Surg Oncol. 2017;24:3683-91.

5. Japanese Gastric Cancer Association. Japanese classification of gastric carcinoma. 15 th. Tokyo: Kanehara; 2017 (in Japanese).

6. Dhar DK, Kubota H, Tachibana M, Kinugasa S, Masunaga R, et al. Prognosis of T4 gastric carcinoma patients: an appraisal of aggressive surgical treatment. J Surg Oncol. 2001;76:278-82.

7. Kobayashi A, Nakagohri T, Konishi M, Inoue K, Takahashi S, et al. Aggressive surgical treatment for T4 gastric cancer. J Gastrointest Surg. 2004;8:464-70.

8. Carboni F, Lepiane P, Santoro R, Lorusso R, Mancini P, et al. Extended multiorgan resection for T4 gastric carcinoma: 25-year experience. J Surg Oncol. 2005;90:95-100.

9. Isozaki H, Tanaka N, Tanigawa N, Okajima K. Prognostic factors in patients with advanced gastric cancer with macroscopic invasion to adjacent organs treated with radical surgery. Gastric Cancer. 2000;3:202-10.

10. Martin RCG II, Jaques DP, Brennan MF, Karpeh M. Extended local resection for advanced gastric cancer: increased survival versus increased morbidity. Ann Surg. 2002;236:159-65.

11. Kodama I, Takamiya H, Mizutani K, Ohta J, Aoyagi K, et al. Gastrectomy with combined resection of other organs for carcinoma of the stomach with invasion to adjacent organs: clinical efficacy in a retrospective study. J Am Coll Surg. 1997;184:16-22.

12. Saito H, Tsujitani S, Maeda Y, Fukuda K, Yamaguchi K, et al. Combined resection of invaded organs in patients with T4 gastric carcinoma. Gastric Cancer. 2001;4:206-11.

13. Kunisaki C, Akiyama H, Nomura M, Matsuda G, Otsuka Y, et al. Surgical outcomes in patients with T4 gastric carcinoma. J Am Coll Surg. 2006;202:223-30.

14. Kim DY, Joo JK, Seo KW, Park YK, Ryu SY, et al. T4 gastric carcinoma: the benefit of non-curative resection. ANZ J Surg. 2006;76:453-7.

15. Min JS, Jin SH, Park S, Kim SB, Bang HY, et al. Prognosis of curatively resected pT4b gastric cancer with respect to invaded organ type. Ann Surg Oncol. 2012;19:494-501.

16. Jeong O, Choi WY, Park YK. Appropriate selection of patients for combined organ resection in cases of gastric carcinoma invading adjacent organs. J Surg Oncol. 2009;100:115-20.

\section{Publisher's Note}

Springer Nature remains neutral with regard to jurisdictional claims in published maps and institutional affiliations. 